\title{
Propofol-Associated Priapism in a Pediatric Patient
}

Journal of Pharmacy Technology

20I5, Vol. 3I (2) 9I-93

(C) The Author(s) 2014

Reprints and permissions:

sagepub.com/journalsPermissions.nav

DOI: 10.1 |77/8755|225|455|419

pharmatech.sagepub.com

(S)SAGE

\author{
Alissa N. Doherty, MD ${ }^{1,2}$ and Paras B. Khandhar, MD, FAAP ${ }^{1,2}$
}

\begin{abstract}
Objective: To report a case of priapism in an adolescent male following the use of propofol for sedation. Case Report: A 13-year-old male with developmental delay was admitted to intensive care secondary to pneumonia and persistent paroxysmal choreoathetosis. In attempt to stop the choreoathetosis and progressive rhabdomyolysis, he was sedated with $50 \mathrm{mg}$ of intravenous propofol, which successfully resolved the choreoathetosis. Within minutes after the injection, he developed priapism, and within I hour it resolved without intervention. Discussion: Priapism was considered to be caused by propofol based on temporal relationship, lack of other medications administered, and complete resolution of symptoms after withdrawal. An objective causality assessment based on the Naranjo adverse drug reaction probability scale suggests propofol is a probable cause of the priapism in our patient. The mechanism of propofol-induced priapism is unknown but likely occurs due to both local and systemic effects. Conclusion: Pediatric clinicians should be aware of this rare side effect, as it requires prompt recognition and potential urologic intervention to alleviate complications.
\end{abstract}

\section{Keywords}

propofol, priapism, pediatric, adverse reaction, side effect

\section{Introduction}

Propofol is a procedural sedative-hypnotic medication used with increasing frequency in pediatrics. The popularity of this medication is likely due to its short duration of action and limited side effect profile. Overall, propofol is well tolerated with minimal adverse reactions in pediatrics. Nevertheless, some side effects such as apnea, seizures, and propofol infusion syndrome have been reported. ${ }^{1}$

We present a case of priapism in a pediatric patient following the use of propofol for sedation in our pediatric intensive care unit (PICU). To our knowledge, only 3 cases of this rare side effect have been reported, 2 of which were in children. ${ }^{2-4}$

\section{Case Report}

A 13-year-old white male with a history of global developmental delay, tracheostomy-dependent respiratory failure, and intermittent choreoathetosis was admitted to the PICU with respiratory distress secondary to pneumonia and persistent paroxysmal choreoathetoid movements.

His home medications included clonidine $0.1 \mathrm{mg}$ every evening, which he was given on arrival to the PICU at 18:28. Other home medications included diazepam and albuterol as needed. In an attempt to decrease the severity of choreoathetosis, he was given $2 \mathrm{mg}$ of lorazepam at 17:30 and 20:58 without success. He was then administered $2 \mathrm{mg}$ of morphine at 21:47, but his movements persisted. He subsequently developed rhabdomyolysis with progressive elevation in creatine kinase level (2426 U/L). An intravenous bolus of $50 \mathrm{mg}$ of propofol, given at 01:30, alleviated his choreoathetosis. As demonstrated above, propofol was given approximately 7 hours after administration of clonidine and 3.5 hours after administration of morphine. Within minutes of the propofol injection, he developed priapism. He received aggressive intravenous hydration with stable urine output. No further propofol was administered. The priapism resolved after approximately 1 hour, without any additional therapy.

\section{Discussion}

Priapism is a pathologic engorgement of the penis in the absence of sexual arousal. ${ }^{5}$ It is classified as a low-flow, ischemic state or a high-flow, nonischemic state. ${ }^{5}$ Low-flow priapism is the more common of the 2 states and is characterized by lack of venous drainage from the corpora

\footnotetext{
'Oakland University William Beaumont School of Medicine, Rochester, MI, USA

${ }^{2}$ Beaumont Children's Hospital, Royal Oak, MI, USA

\section{Corresponding Author:}

Alissa N. Doherty, MD, Beaumont Children's Hospital, Department of Pediatrics, 360I West I 3 Mile Road, Royal Oak, MI 48073, USA.

Email: anordin@med.umich.edu
} 
cavernosa, vascular stasis, acidosis, and tissue hypoxia. This is a medical emergency, as irreversible damage and impotence may occur if the priapism is not corrected within 24 hours. ${ }^{5,6}$ High-flow priapism is characterized by disruption of arterial vasculature supplying the penis and increased arterial inflow into the sinusoidal space of the corpora cavernosa. High-flow priapism is most commonly due to penile or perineal trauma, but a few cases have been reported in patients with sickle cell disease and leukemia. ${ }^{7}$ High-flow priapism is typically self-limited and there are no ischemic complications.

In addition to hematologic disorders, malignancies, and trauma, priapism has followed the use of certain medications. Drug-induced priapism is most commonly due to low-flow pathology and requires prompt recognition and treatment. ${ }^{5}$ Associated medications include antihypertensives, especially guanethidine, prazosin, and hydralazine, and psychotropics, commonly chlorpromazine, trazodone, and thioridazine. ${ }^{5}$ Although these are the most common medications, others have also been associated with this side effect. As mentioned previously, our patient is regularly taking clonidine daily. Clonidine decreases peripheral vascular resistance by acting as an $\alpha$-adrenergic agonist and reducing sympathetic outflow; therefore, it may potentiate central nervous system depressive effects of sedative medications. ${ }^{8}$ Clonidine is a relatively fast-acting medication reaching peak plasma levels at approximately 1 to 3 hours and has a biphasic elimination pattern of approximately 20 minutes with a terminal half-life of 12 to 16 hours. $^{8}$ Our patient takes clonidine daily and has not had any previous episodes of priapism; therefore, clonidine is unlikely the inciting medication. As our patient was given propofol approximately 7 hours after clonidine, it is possible that clonidine may have potentiated the effect of propofol leading to priapism, although due to the relatively low dose, lack of renal failure, and lack of significant temporal relationship, it is unlikely that there was a drug-drug interaction leading to priapism in our patient.

We believe that our patient's priapism was caused by propofol because of the temporal relationship of symptoms, lack of other medications administered at the time of the event, and the complete resolution of symptoms after withdrawal of propofol. Naranjo et al created a probability scale to determine if an adverse reaction is due to a specific medication. ${ }^{9}$ Based on this scale, propofol is a probable cause of the priapism in our patient. Directly following administration of propofol, our patient developed priapism, and within 1 hour after withdrawal, the priapism resolved without intervention. Because we did not subject our patient to a rechallenge with propofol, we could not demonstrate a dose-dependent relationship. Nevertheless, propofol remains a probable cause of his priapism.

As previously mentioned, 3 other cases of propofol-associated priapism have been described in the literature. ${ }^{2-4}$ Vesta et al describe a case of priapism in a 17-year-old male undergoing cardiac ablation after he was given propofol for sedation and again on rechallenge when given propofol for transesophageal echocardiogram the following day. ${ }^{2}$ Of note, this patient was given tramadol prior to the first procedure and fentanyl immediately prior to the second procedure. Fuentes et al describe a case of propofol-associated priapism in a 7-year-old child with cerebral palsy after receiving propofol for a surgical procedure. ${ }^{3}$ Of note, this patient was also given fentanyl for induction of sedation. He required surgical intervention for resolution of priapism. Balamurgan et al describe a case of propofol-associated priapism in a 25 -yearold male while undergoing closed reduction of a dislocated shoulder. ${ }^{4}$ He had recurrence of his dislocation 3 days later and underwent sedation with propofol and again had priapism shortly after infusion. Of note, the second administration of propofol occurred shortly after an injection of fentanyl. Interestingly, each patient in the previous cases, as well as our patient, was administered an opiate medication in addition to propofol. Opiates are mu-receptor agonists and cause analgesia and central nervous system and respiratory depression, as well as decreased peripheral vascular resistance. ${ }^{10}$ Perhaps priapism may result from potentiated effects of propofol and opiates when administered together. The relationship between propofol and opiates in the aforementioned case reports is unclear, although it is an interesting finding that warrants further investigation.

Historically, priapism has been described in patients receiving total parenteral nutrition (TPN) containing 20\% concentration of intralipid fat emulsion but not in those receiving the $10 \%$ concentration of fat. ${ }^{11,12}$ Although the cause of priapism in those cases is not completely clear, the postulated mechanisms include fat embolism, increased coagulability, and increased platelet function. ${ }^{5,11,13}$ Propofol is formulated as an oil-in-water emulsion, similar to TPN, and contains approximately $10 \%$ fat. ${ }^{12,14}$ Due to this significant difference, it is unlikely that the fat is the underlying cause of priapism in our patient. In addition, the total amount of fat delivered from a single dose of propofol is far lower than that delivered to patients receiving TPN.

The exact mechanism by which propofol may cause priapism is unknown. Sweni et al hypothesized that propofol may cause priapism by acting both centrally in affecting NMDA receptors, GABA, serotonin, and dopamine, and locally by increasing nitric oxide smooth muscle relaxation. ${ }^{15}$ Regardless of the mechanism, our patient's priapism cannot be plausibly explained by anything but propofol. He had no other underlying disease, temporally related medications, or other factors that could explain his symptoms.

\section{Conclusion}

We report a case of propofol-associated priapism in a 13-year-old patient. If not medically or surgically corrected, this uncommon side effect can have disastrous 
consequences. Given the worldwide use of propofol in pediatrics for sedation and anesthesia, clinicians should be aware of this potential side effect in males.

\section{Authors' Note}

This article was presented as a poster presentation at the Pediatric Academic Societies national meeting in Washington, DC, in May 2013.

\section{Declaration of Conflicting Interests}

The author(s) declared no potential conflicts of interest with respect to the research, authorship, and/or publication of this article.

\section{Funding}

The author(s) received no financial support for the research, authorship, and/or publication of this article.

\section{References}

1. Marik PE. Propofol: therapeutic indications and side effects. Curr Pharm Des. 2004;10:3639-3649.

2. Vesta K, Martina S, Kozlowski E. Propofol-induced priapism: a case confirmed with rechallenge. Ann Pharmocother. 2006;40:980-982.

3. Fuentes E, Garcia S, Garrido M, Lorenzo C, Iglesias J, Sola J. Successful treatment of propofol-induced priapism with distal glans to corpus cavernosal shunt. Urology. 2009;74:113-115.

4. Balamurgan N, Ganapathysubramanian B, Senthilkumaran S, Shah S, Thurumalaikolundusubramanian P. Propofol and priapism. Indian J Pharmacol. 2010;42:238-239.
5. Sadeghi-Nejad H, Dogra V, Seftel A, Mohamed M. Priapism. Radiol Clin North Am. 2004;42:427-443.

6. American Urological Association, Inc. Guideline on the Management of Priapism. Linthicum, MD: American Urological Association, Inc. https://www.auanet.org/common/ pdf/education/clinical-guidance/Priapism.pdf. Published 2003. Accessed September 9, 2014.

7. Corbetta J, Duran V, Burek C, et al. High flow priapism: diagnosis and treatment in pediatric population. Pediatr Surg Int 2011;27:1217-1221.

8. Clonidine [Package insert]. Mexico City, Mexico: Boehringer Ingleheim Pharmaceuticals; 2011.

9. Naranjo C, Busto U, Sellers E, et al. A method for estimating the probability of adverse drug reactions. Clin Pharmacol Ther. 1981;30:239-245.

10. Morphine [Package insert]. Columbus, OH: Roxane Laboratories; 2011.

11. Ekstrom B, Olsson AM. Priapism in patients treated with total parenteral nutrition. Br J Urol. 1987;59:170-171.

12. Diprivan (propofol) [Package insert]. Capanago, Italy: AstraZeneca Pharmaceuticals; July 2004.

13. Klein E, Montague D, Steiger E. Priapism associated with the use of intravenous fat emulsion: case reports and postulated pathogenesis. J Urol. 1985;133:857-859.

14. Roth M, Martin A, Katz J. Nutritional implications of prolonged propofol use. Am J Health Syst Pharm. 1997;54: 694-695.

15. Sweni S, Meenakshisundaram R, Senthilkumaran S, Thirumalaikolundusubramanian P. Propofol's derivative: a potential drug for erectile dysfunction? Med Hypotheses. 2011;77:668-670. 